\title{
A Simplified C-Logit Stochastic User Equilibrium Model on Bimodal Transportation Network
}

\author{
Bingquan Liu $\mathbb{D}^{1}{ }^{1}$ Yonghong Zhang, ${ }^{1}$ and Wei $\mathrm{Du}^{2}$ \\ ${ }^{1}$ School of Mathematics and Statistics, Weinan Normal University, Weinan 714099, China \\ ${ }^{2}$ School of Transportation, Nantong University, Nantong 226019, China \\ Correspondence should be addressed to Bingquan Liu; 215733251@qq.com
}

Received 27 October 2019; Accepted 21 March 2020; Published 25 April 2020

Academic Editor: Massimiliano Ferrara

Copyright ( 2020 Bingquan Liu et al. This is an open access article distributed under the Creative Commons Attribution License, which permits unrestricted use, distribution, and reproduction in any medium, provided the original work is properly cited.

This paper investigates the C-logit stochastic user equilibrium (SUE) problem on a bimodal transportation network with road and rail travel modes. The C-logit model captures the overlapping effect among the different paths via commonality factors; sequentially, it has ability to obtain a more realistic traffic flow distribution pattern. In this paper, when we redefine the link travel cost functions and employ a binary Logit model for the mode split, the bimodal C-logit SUE model can be simplified into an unconstrained nonlinear mathematical programming formulation. Such model is verified to satisfy the bimodal C-logit SUE conditions at its stationary point and can be solved by existing algorithms. So, the simplified model can be convenient to be used on the general bimodal transportation network.

\section{Introduction}

It is widely recognized that traffic congestion is a main threat to the sustainable urban transportation system. The increasing emissions and air pollution problems induced by congestion are severely harmful to the environment and human physical and mental health. To mitigate the increasing traffic congestion, the transportation sectors in many dense metropolises spare no effort to develop the public transport system. Especially, the rail network, an indispensable part of public transport, is more space efficient to meet the expanded traffic demand with trivial influence on the travel cost and environmental pollution. Owing to its convenience, serviceability and punctuality, rail transportation is deemed to the backbone of the public transport system, and it facilitates the development of the urban regions [1]. Thus, the simplex road network in urban areas has been converted into the complex hybrid network with road and rail. In this paper, for brevity, the bimodal transportation network is specified for road mode and rail mode.

On the road transportation network, the general assumption of the traffic assignment model is the deterministic user equilibrium (DUE) principle; all travelers have equal and minimum path travel cost and cannot improve their path travel cost by unilaterally changing paths [2]. The DUE principle always supposes that all travelers can perfectly acknowledge the network information and consistently choose paths to minimize their travel cost. The assumption is so rigorous for travelers that it cannot hold on the realistic network. The principle of SUE proposed by Daganzo and Sheffi can further relax the assumption and be stated that all travelers cannot improve their perceived travel cost by unilaterally changing paths [3]. Based on the SUE principle, the perceived travel cost can be expressed by the actual travel cost and a random error term as follows:

$$
C_{k}^{r s}=c_{k}^{r s}+\xi_{k}^{r s}, \quad \forall k \in K^{r s}, r \in R, s \in S,
$$

and the expected perceived travel cost between OD pair $(r, s)$ is defined by

$$
\pi^{r s}=E\left(\min _{k \in K^{r s}}\left(C_{k}^{r s} \mid c_{k}^{r s}+\xi_{k}^{r s}\right)\right) .
$$

The notations used in formulations (1) and (2) are shown in Table 1.

If the random error terms follow independently and identically Gumbel distribution, the SUE model is the 
TABLE 1: Basic notations.

\begin{tabular}{ll}
\hline$R$ & Set of origins between the OD pairs \\
$\bar{A}$ & Set of destinations between the OD pairs \\
$K^{r s}$ & Set of links tolled on the road network \\
$\widehat{K}^{r s}$ & Set of noncyclic paths connecting the OD pair $(r, s)$ on the road network \\
$C_{k}^{r s}$ & The set of noncyclic paths connecting the OD pair $(r, s)$ on the rail network \\
$c_{k}^{r s}$ & perceived path travel cost on path $k\left(k \in K^{r s}\right)$ on the road network \\
$\xi_{k}^{r s}$ & Actual path travel cost on path $k$ on the road network \\
$\pi^{r s}$ & Random error term on path $k$ on the road network, and $E\left(\xi_{k}^{r s}\right)=0$ \\
$\widehat{\pi}^{r s}$ & Expected perceived travel cost between OD pair $(r, s)$ on the road network \\
$\mathrm{CF}_{k}^{r s}$ & Constant travel cost between OD pair $(r, s)$ on the rail network \\
$p_{k}^{r s}$ & Commonality factor on path $k$ \\
$f_{k}^{r s}$ & Path choice probability of path $k$ on the road network \\
$x_{a}$ & Flow on path $k, k \in K^{r s} \cup \widehat{K}^{r s}, \mathbf{f}=\left(\ldots, f_{k}^{r s}, \ldots\right)^{T}$ \\
$t_{a}\left(x_{a}\right)$ & Flow on link $a, a \in A \cup \widehat{A}, \mathbf{x}=\left(\ldots, x_{a}, \ldots\right)^{T}$ \\
$q_{r s}$ & Travel cost on link $a, a \in A \cup \widehat{A}, \mathbf{t}(\mathbf{x})=\left(\ldots, t_{a}\left(x_{a}\right), \ldots\right)^{T}$ \\
$\widehat{q}_{r s}$ & Demand between OD pair $(r, s)$ on the road network \\
$Q_{r s}$ & Demand between OD pair $(r, s)$ on the rail network \\
$\Delta=\left[\delta_{a k}^{r s}\right]$ & Total demand between OD pair $(r, s)$ on the bimodal network, $Q_{r s}=q_{r s}+\widehat{q}_{r s}, \mathbf{Q}=\left(\ldots, Q_{r s}, \ldots\right)^{T}$ \\
$\Gamma=\left[\gamma_{k}^{r s}\right]$ & Link-path matrix, $\delta_{a k}^{r s}=1$ if path $k$ uses link $a$, and 0 otherwise \\
$\theta$ & OD pair-path matrix, $\gamma_{k}^{r s}=1$ if path $k$ connects the OD pair $(r, s)$, and 0 otherwise \\
$\alpha$ & Perception parameter, describe the degree of dispersion of the network, $\theta>0$ \\
\hline
\end{tabular}

popular multinomial logit (MNL) model. Under the conditions of additive path cost and separable link cost, the MNL is equivalent to a convex minimization formulation proposed by Fisk [4]. If the random error terms follow the normal distribution, the SUE model is the multinomial Probit (MNP) model. The MNL possesses a closed-form expression of path choice behavior but inheres in the drawback of independence of irrelevant alternative (IIA) property, that is, it is unable to account for overlapping effect and covariance among the different travel path cost $[5,6]$. The MNP model overcomes such a drawback by taking the path cost covariance into account, but there are relatively few studies in the area of Probit SUE, such as Lam and $\mathrm{Xu}$ [7], Meng et al. [8], Meng and Liu [9], Cantarella et al. [10], and Jing et al. [11] since it does not possess a explicit closed-form expression and behavioural interpretation. Generally, the MNP model describes travelers' path choice behaviors by using some simulation-based methods $[1,9,12]$. The methods are computationally time consuming due to the requirements of high calibration and specification efforts when numerous paths are available on a road network. To overcome the drawbacks of MNL and MNP, many improvements of MNL have been submitted recently where an outstanding example is C-logit SUE model proposed by Cascetta et al. [13] and further analyzed and investigated by Zhou et al. [14]. The C-logit SUE model has ability to deal with the overlapping effect between the paths by adding a commonality factor into the path cost function. Chen et al. investigated the scaling effect and overlapping problem in a route choice context using the $\mathrm{C}$-logit and other correlative logit model to explicitly account for the congestion effect. They demonstrated that the scaling effect and overlapping problem made a significant impact on the equilibrium flow distribution $[15,16]$.

Zhou et al. investigated the C-logit SUE models on the road transportation network [14]. Two versions of commonality factors are provided in their models, flow-independent and flow-dependent, i.e., evaluating the commonality factors based on the free-flow travel cost and current path travel cost, respectively. The corresponding SUE is called the length-based SUE or congestion-based SUE. In their study, a mathematical programming formulation and two variational inequality (VI) formulations are proposed, respectively, for the length-based SUE and the congestion-based SUE. However, this paper only takes length-based C-logit SUE into account on the bimodal transportation network since the congestion-based C-logit model can be similarly analyzed. Under the general assumptions, when the link cost function is redefined and a binary logit model is used for the mode-split, the length-based bimodal C-logit SUE model is transformed into an unconstrained nonlinear programming formulation. The simplified unconstrained optimization model satisfies the bimodal C-logit SUE conditions at its stationary point. Particularly, the link flowbased stationary point is a unique global minimum point for the strictly monotonous link cost function in the formulation. Differing from the previous research studies, our model integrates the bimodal network into the single network with fixed traffic demand; hence, it can be analyzed easily and be solved by some existing algorithms.

This paper is organized as follows. Section 2 presents the basic notations and the bimodal C-logit SUE models and explains the solution characteristics. Section 3 proposes two second-best congestion pricing models and their respective solution algorithms. Section 4 concludes the paper and recommends further studies.

\section{Bimodal C-Logit SUE Problem}

2.1. Basic Notations and General Assumptions. Given a bimodal transportation network represented by a strongly connected graph $G=(N, A \cup \widehat{A})$, where $N$ is the set of nodes, $A$ is the set of links on the road network, and $\widehat{A}$ is the set of links on the rail network. Every path on the rail network is taken as a link. The notations used in this paper are summarized in Table 1. 
To simplify the exposition, the following four assumptions, first, are made throughout the paper without loss of generality:

(A1) The link cost function on the road network is separable, nonnegative, strictly monotonic, and continuously differentiable

(A2) For an OD pair without rail service, it may be set a dummy rail link with infinite travel cost

(A3) Any two used rail paths have identical constant cost between the same OD pair, and travel demand is assigned averagely into every rail path

(A4) Any two rail paths do not exist overlapping effect on the rail network, i.e., $\mathrm{CF}_{a}^{r s}=0, \forall a \in \widehat{A}$

Remark 1. Generally, there exists at most a rail path between an OD pair; however, it is possible for two or more rail paths to exist between an OD pair, especially for the region with higher trip productions and attractions. As shown in Figure 1, A, B, and C are three regions with higher trip productions, and $\mathrm{D}$ is a region with higher trip attraction. There may be two rail paths between the OD pair $\mathrm{AD}$. Based on assumption $\mathrm{A} 3$, each rail path flow $f_{\mathrm{ABD}}=f_{\mathrm{ACD}}=(1 / 2) \hat{q}_{\mathrm{AD}}$, where $\hat{q}_{\mathrm{AD}}$ denotes the travel demand between the OD pair $\mathrm{AD}$.

Remark 2. If there exist two rail paths with different travel costs between an OD pair, following the SUE principle, the path with higher travel cost is not used and can be deleted from the rail network.

2.2. Length-Based Bimodal C-Logit SUE Model. The commonality factor employed by Zhou et al. is formulated as the following form [14]:

$$
\mathrm{CF}_{k}^{r s}=\kappa \ln \sum_{l \in K^{r s}}\left(\frac{L_{l k}}{\sqrt{L_{k}} \sqrt{L_{l}}}\right), \quad \forall k \in K^{r s}, r \in R, s \in S,
$$

where $L_{l k}$ denotes the length of links common to path $l$ and $k, L_{l}$ and $L_{k}$ is the overall length of path $l$ and $k$ respectively, and $\kappa$ is a parameter. The length can represent both link(path-) free flow cost and current flow cost.

The C-logit SUE model overcomes the deficiencies of the MNL path choice behavior and accounts for the overlapping effect among the paths between every OD pair. The closedform path choice probability of the C-logit model is given below:

$$
p_{k}^{r s}=\frac{e^{-\theta\left(c_{k}^{r s}+\mathrm{CF}_{k}^{r s}\right)}}{\sum_{l \in K^{r s}} e^{-\theta\left(c_{l}^{r s}+\mathrm{CF}_{l}^{r s}\right)}}, \quad \forall k \in K^{r s}, r \in R, s \in S .
$$

The C-logit SUE condition on the road network is expressed as follows:

$$
f_{k}^{r s}=q_{r s} p_{k}^{r s}, \quad \forall k \in K^{r s}, r \in R, s \in S .
$$

With the C-logit-based path choice probability (4), the expected perceived travel cost between OD pair $(r, s)$ can be explicitly expressed as follows:

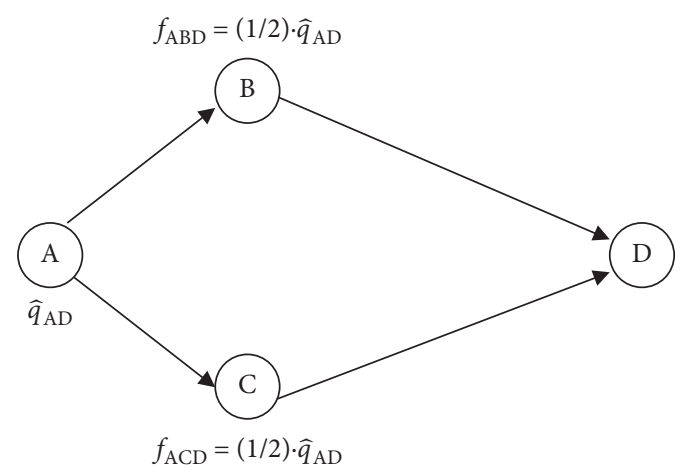

Figure 1: Graphical representation of two rail paths.

$\pi^{r s}=E\left(\min \left(C_{k \in K^{r s}}^{r s}+\mathrm{CF}_{k}^{r s}\right)\right)=-\frac{1}{\theta} \ln \sum_{k \in K^{r s}} e^{-\theta\left(c_{k}^{r s}+C F_{k}^{r s}\right)}, \quad \forall r \in R, s \in S$.

Suppose the expected perceived travel cost $\hat{\pi}^{r s}$ between OD pair $(r, s)$ is a constant and the binary Logit model is used for the mode split. Mode equilibrium can be explicitly expressed as follows:

$$
\begin{aligned}
& q_{r s}=\frac{Q_{r s}}{\left(1+e^{\alpha\left(\pi^{r s}-\widehat{\pi}^{r s}\right)}\right)}, \\
& \hat{q}_{r s}=\frac{Q_{r s}}{\left(1+e^{\alpha\left(\widehat{\pi}^{r s}-\pi^{r s}\right)}\right)},
\end{aligned}
$$

where $q_{r s}$ and $\hat{q}_{r s}$ are the demand between OD pair $(r, s)$ on the road network and the rail network, respectively.

With assumptions (A1)-(A4), length-based bimodal C-logit SUE model can be formulated as follows:

$$
\begin{aligned}
& \min _{f} Z(f)= \sum_{a \in A} \int_{0}^{v_{a}} t_{a}(\omega) \mathrm{d} \omega+\frac{1}{\theta} \sum_{r \in R} \sum_{s \in S} \sum_{k \in K^{r s}} f_{k}^{r s}\left(\ln f_{k}^{r s}-1\right) \\
&+\sum_{r \in R} \sum_{s \in S} \sum_{k \in K^{r s}} f_{k}^{r s} \mathrm{CF}_{k}^{r s}+\sum_{r \in R} \sum_{s \in S} \widehat{q}_{r s} \widehat{\pi}^{r s} \\
&+\frac{1}{\alpha} \sum_{r \in R} \sum_{s \in S} q_{r s}\left(\ln \frac{q_{r s}}{Q_{r s}}-1\right)+\frac{1}{\alpha} \sum_{r \in R} \sum_{s \in S} \widehat{q}_{r s}\left(\ln \frac{\widehat{q}_{r s}}{Q_{r s}}-1\right), \\
& \text { s.t. } \sum_{k \in K^{r s}} f_{k}^{r s}=q_{r s}, \quad \forall r, s, \\
& q_{r s}+\widehat{q}_{r s}=Q_{r s}, \quad \forall r, s, \\
& x_{a}=\sum_{r \in R} \sum_{s \in S} \sum_{k \in K^{r s}} f_{k}^{r s} r_{a k}^{r s}, \quad \forall a \in A, \\
& f_{k}^{r s} \geq 0, \forall k \in K^{r s}, \quad \forall r, s .
\end{aligned}
$$

Proposition 1. The minimization formulation (9) satisfies the bimodal C-logit SUE conditions (4) and (5) and (7) and (8) at its stationary point (KKT point). 
Proof. Based on $f>0$, the Lagrange function of formulation (9) can be simplified into

$$
\begin{aligned}
L(f, q, \hat{q}, \dot{\mu}, \pi)= & Z(f)-\sum_{r \in R} \sum_{s \in S} \dot{\mu}_{r s}\left(q_{r s}+\widehat{q}_{r s}-Q_{r s}\right) \\
& -\sum_{r \in R} \sum_{s \in S} \pi_{r s}\left(\sum_{k \in K^{r s}} f_{k}^{r s}-q_{r s}\right),
\end{aligned}
$$

where $\dot{\mu}$ and $\pi$ are the Lagrange multiplier; so

$$
\begin{aligned}
\frac{\partial L}{\partial f_{k}^{r s}} & =\ln f_{k}^{r s}+\mathrm{CF}_{k}^{r s}-\pi_{r s}+\sum_{a \in A} t_{a}\left(v_{a}\right) \delta_{a k}^{r s}=0, \\
\frac{\partial L}{\partial q_{r s}} & =\frac{1}{\alpha} \ln \frac{q_{r s}}{Q_{r s}}-\dot{\mu}_{r s}+\pi_{r s}=0 \\
\frac{\partial L}{\partial \hat{q}_{r s}} & =\frac{1}{\alpha} \ln \frac{\widehat{q}_{r s}}{Q_{r s}}-\dot{\mu}_{r s}+\widehat{\pi}_{r s}=0
\end{aligned}
$$

$$
\forall r, s .
$$

The abovementioned system of equations can obtain the following formulation easily:

$$
\begin{aligned}
& f_{k}^{r s}=q_{r s} \frac{e^{-\theta\left(c_{k}^{r s}+\mathrm{CF}_{k}^{r s}\right)}}{\sum_{l \in K^{r s}} e^{-\theta\left(c_{l}^{r s}+\mathrm{CF}_{l}^{r s}\right)},} \\
& q_{r s}=\frac{Q_{r s}}{\left(1+e^{\left.\alpha\left(\pi^{r s}-\widetilde{\pi}^{r s}\right)\right)},\right.} \\
& \hat{q}_{r s}=\frac{Q_{r s}}{\left(1+e^{\left.\alpha\left(\widetilde{\pi}^{s}-\pi^{r s}\right)\right)}\right.} .
\end{aligned}
$$

$$
t_{a}(\omega)= \begin{cases}t_{a}(\omega), & \text { if } a \in A, \\ \widehat{\pi}^{r s}+\left(\frac{1}{\theta}-\frac{1}{\alpha}\right)\left(\ln \left(Q_{r s}-\left|\widehat{K}^{r s}\right| \omega\right)-\ln \omega\right)+\frac{1}{\alpha} \ln \left|\widehat{K}^{r s}\right|-C F_{a}^{r s}, & \text { if } a \in \widehat{A} .\end{cases}
$$

That means that model (9) satisfies the bimodal C-logit SUE conditions (4) and (5) and (7) and (8) at its stationary point (KKT point).

This completes the proof.

\section{A Simplified Length-Based C-Logit SUE Model}

According to model (9), both C-logit SUE conditions (4) and (5) and mode equilibrium conditions (7) and (8) on the bimodal network can be followed, but the objective function is complicated and there are huge amounts of constraints in formulation (9). Hence, we consider a simplified model below.

Based on the abovementioned assumptions (A1)-(A4), the length-based C-logit SUE model can be transformed into the following unconstrained minimization formulation:

$$
\begin{aligned}
\min _{\mathbf{f}} \bar{Z}(\mathbf{f})= & \frac{1}{\theta} \sum_{r \in R} \sum_{s \in S} Q_{r s} \ln \sum_{k \in K^{r s} \cup \widehat{K}} e^{-\theta\left(c_{k}^{r s}+\mathrm{CF}_{k}^{r s}\right)} \\
& +\sum_{a \in A \cup \widehat{A}} x_{a} t_{a}-\sum_{a \in A \cup \widehat{A}} \int_{0}^{x_{a}} t_{a}(\omega) \mathrm{d} \omega,
\end{aligned}
$$

where
$\widehat{A}=\cup_{r \in R} \bigcup_{s \in S} \widehat{K}^{r s}$ denotes to take the rail paths as links on the bimodal transportation network.

Note that, after redefining the link travel cost function, (13) is similar to the unconstrained minimization formulation proposed by Daganzo and Sheffi [3], Zhou et al. [17], or Xie and Travis Waller [18] on the road network. However, models (13) and (14) integrate the bimodal network into the single network with fixed traffic demand; hence, it differs form previous formulations and can be analyzed and solved by some existing algorithms easily. Furthermore, it possesses an important mathematical property as stated in the following proposition.

Proposition 2. Under assumptions (A1)-(A4), the unconstrained minimization formulations (13) and (14) satisfies the bimodal C-logit SUE conditions (4) and (5) and (7) and(8) and the link flow-based stationary point is the unique global minimum point. 
Proof. Given $\quad c_{k}^{r s}=\sum_{a \in A \cup \widehat{A}^{t}} \quad\left(x_{a}\right) \delta_{a k}^{r s}, x_{a}=\sum_{r \in R} \sum_{s \in S}$ $\sum_{k \in K^{r s} \cup \widehat{K}^{r s}} f_{k}^{r s} \delta_{a k}^{r s}$, the first-order derivatives of $\bar{Z}(\mathbf{f})$ with respect to any path flow variable $f_{k}^{\text {od }}$ can be expressed as follows:

$$
\begin{gathered}
\frac{\partial \bar{Z}(f)}{\partial f_{k}^{o d}}=-\sum_{r \in R} \sum_{s \in S} Q_{r s} \sum_{l \in K^{r s} \cup} \frac{e^{-\theta\left(c_{l}^{r s}+\mathrm{CF}_{l}^{r s}\right)}}{\sum_{h \in K^{r s} \cup} \widehat{K}^{r s} e^{-\theta\left(c_{h}^{r s}+\mathrm{CF}_{h}^{r s}\right)}} \sum_{a \in A \cup \widehat{A}} \delta_{a l}^{r s} \delta_{a k}^{o d} \frac{\partial t_{a}}{\partial x_{a}}+\sum_{a \in A \cup \widehat{A}} x_{a} \frac{\partial t_{a}}{\partial x_{a}} \delta_{a k}^{o d} \\
=-\sum_{a \in A \cup \widehat{A}} \delta_{a k}^{o d} \frac{\partial t_{a}}{\partial x_{a}} \sum_{r \in R} \sum_{s \in S} \sum_{s \in K^{r s} \cup \widehat{K}^{r s}} \delta_{a l}^{r s}\left(Q_{r s} \frac{e^{-\theta\left(c_{l}^{r s}+\mathrm{CF}_{l}^{r s}\right)}}{\left.\sum_{h \in K^{r s} \cup \widehat{K}^{r s} e^{-\theta\left(c_{h}^{r s}+\mathrm{CF}_{h}^{r s}\right)}}-f_{l}^{r s}\right),}\right. \\
\forall k \in K^{o d} \cup \widehat{K}^{o d}, o \in R, d \in S .
\end{gathered}
$$

Setting $\left(\partial \bar{Z}(\mathbf{f}) / \partial f_{k}^{o d}\right)=0$, we have the following fixed point equation including a path flow-based stationary point:

$$
f_{k}^{r s}=Q_{r s} \frac{e^{-\theta\left(c_{k}^{r s}+\mathrm{CF}_{k}^{r s}\right)}}{\sum_{l \in K^{r s} \cup \widehat{K}^{r s} e^{-\theta\left(c_{l}^{r s}+\mathrm{CF}_{l}^{r s}\right)}}} \quad \forall k \in K^{r s} \cup \widehat{K}^{r s}, r \in R, s \in S .
$$

Let $\left(\partial \bar{Z}(\mathbf{f}) / \partial x_{a}\right)=0$, and we have

$$
x_{a}=\sum_{r \in R} \sum_{s \in S} \sum_{l \in K^{r s} \cup}{\widehat{K}^{r s}}_{r s} \frac{\exp \left(-\theta\left(c_{l}^{r s}+\mathrm{CF}_{l}^{r s}\right)\right)}{\sum_{h \in K^{r s} \cup \widehat{K}^{r s}} \exp \left(-\theta\left(c_{h}^{r s}+\mathrm{CF}_{h}^{r s}\right)\right)} \delta_{a l}^{r s} \text {. }
$$

Sheffi investigated and analyzed the unconstrained minimization formulation proposed by Daganzo and showed that the SUE link flow solution was the unique global minimum point for the strictly monotonous link cost function [19]. Similarly, model (13) has the same characteristic when the link cost function (14) is strictly monotonous. This shows that the link flow-based stationary point $\mathbf{x}^{*}$ is the unique global minimum point. From formulation (16), we can also obtain a unique SUE path flow solution $\mathbf{f}^{*}$ in the fixed equation.
If path $k$ is the rail path $\left(k \in \widehat{K}^{r s}\right)$, we have

$$
\begin{aligned}
\widehat{q}_{r s} & =\left|\widehat{K}^{r s}\right| f_{k}^{r s}=Q_{r s} \frac{\left|\widehat{K}^{r s}\right| e^{-\theta t_{r s}}}{\sum_{l \in K^{r s}} e^{-\theta\left(c_{l}^{r s}+C F_{l}^{r s}\right)}+\left|\widehat{K}^{r s}\right| e^{-\theta t_{r s}}} \\
& =Q_{r s} \frac{\left|\widehat{K}^{r s}\right| e^{-\theta t_{r s}}}{e^{-\theta \pi^{r s}}+\left|\widehat{K}^{r s}\right| e^{-\theta t_{r s}}} .
\end{aligned}
$$

Substituting $t_{r s}$ into $\left|\widehat{K}^{r s}\right| e^{-\theta t_{r s}}$, yields

$$
\left|\widehat{K}^{r s}\right| e^{-\theta t_{r s}}=\widehat{q}_{r s}^{1-(\theta / \alpha)} e^{-\theta \widehat{\pi}^{r s}}\left(Q_{r s}-\widehat{q}_{r s}\right)^{(\theta / \alpha)-1}
$$

Substituting $\left|\widehat{K}^{r s}\right| e^{-\theta t_{r s}}$ into right-hand side of equation (18), we have

$$
\begin{aligned}
& \widehat{q}_{r s}=\frac{Q_{r s}}{\left(1+e^{\alpha\left(\hat{\pi}^{r s}-\pi^{r s}\right)}\right)}, \\
& q_{r s}=\frac{Q_{r s}}{\left(1+e^{\alpha\left(\pi^{r s}-\hat{\pi}^{r s}\right)}\right)} .
\end{aligned}
$$

If path $k$ is the road path $\left(k \in K^{r s}\right)$, we have

$$
\begin{aligned}
f_{k}^{r s} & =Q_{r s} \frac{e^{-\theta\left(c_{k}^{r s}+\mathrm{CF}_{k}^{r s}\right)}}{\sum_{l \in K^{r s}} e^{-\theta\left(c_{l}^{r s}+\mathrm{CF}_{l}^{r s}\right)}+\left(\left(Q_{r s}-q_{r s}\right) / q_{r s}\right) \sum_{l \in K^{r s}} e^{-\theta\left(c_{l}^{r s}+\mathrm{CF}_{l}^{r s}\right)}} \\
& =q_{r s} \frac{e^{-\theta\left(c_{k}^{r s}+\mathrm{CF}_{k}^{r s}\right)}}{\sum_{l \in K^{r s}} e^{-\theta\left(c_{l}^{r s}+\mathrm{CF}_{l}^{r s}\right)} .}
\end{aligned}
$$

This means that models (13) and (14) satisfy the bimodal C-logit SUE conditions (4)-(8) at its stationary point.

This completes the proof.

Formulations (13) and (14) can reduce to Daganzo's SUE problem, if only the road network is considered and may be solved by the general unconstrained minimization methods, such as the Newton-type method and conjugate gradient method [20]. So, the simplified model can be convenient to be used on the general bimodal transportation network.

\section{A Simplified Congestion-Based Bimodal C-Logit SUE Model}

The congestion-based C-logit SUE model cannot be formulated as an optimization problem. Instead, Zhou et al. proposed two VI formulations for the C-Logit SUE model with fix demand on the road network [14]. When the link cost function (14) is employed, the congestion-based C-Logit SUE model can also be formulated as a VI problem as follows: 


$$
\boldsymbol{\eta}\left(\mathbf{f}^{*}\right)^{\mathrm{T}}\left(\mathbf{f}-\mathbf{f}^{*}\right) \geq \mathbf{0}, \quad \forall \mathbf{f} \in\{\Gamma \mathbf{f}=\mathbf{Q}, \mathbf{f} \geq \mathbf{0}\},
$$

where

$$
\boldsymbol{\eta}(\mathbf{f})=\left(\ldots, \eta_{k}^{r s}(\mathbf{f}), \ldots\right)^{T}, \eta_{k}^{r s}(\mathbf{f})=c_{k}^{r s}+\frac{1}{\theta} \ln f_{k}^{r s}+c f_{k}^{r s} .
$$

Proposition 3. Under the assumptions of Proposition 2 and the definition of the link cost function (14), the VI formulations (22) and (23) satisfy the bimodal C-Logit SUE conditions (4)-(8).

Proof. The Lagrangian function of the VI formulations (22) and (23) is expressed as follows:

$$
L(\mathbf{f}, \boldsymbol{\mu})=\boldsymbol{\eta}\left(\mathbf{f}^{*}\right) \mathbf{f}-\sum_{r \in R} \sum_{s \in S} \mu^{r s}\left(\sum_{k \in K^{r s} \cup \widehat{K}^{r s}} f_{k}^{r s}-Q_{r s}\right),
$$

where $\boldsymbol{\mu}=\left(\ldots, \mu^{r s}, \ldots\right)^{T}$ is the vector of Lagrangian multiplier. From the KKT conditions, we have

$$
\begin{aligned}
& \frac{\partial L(\mathbf{f}, \boldsymbol{\mu})}{\partial f_{k}^{r s}}=\eta_{k}^{r s}\left(f^{*}\right)-\mu^{r s}=0, \\
& \frac{\partial L(\mathbf{f}, \boldsymbol{\mu})}{\partial \mu^{r s}}=-\sum_{k \in K^{r s} \cup} f_{\widehat{K}^{r s}}^{r s}+Q_{r s}=0 .
\end{aligned}
$$
yields

Substituting formulation (23) into formulation

$$
\begin{aligned}
& f_{k}^{r s}=e^{\theta \mu^{r s}} e^{\left(-\theta c_{k}^{r s}+c f_{k}^{r s}\right)}, \\
& Q_{r s}=e^{\theta \mu^{r s}} \sum_{k \in K^{r s} \cup \widehat{K}^{r s}} e^{-\theta\left(c_{k}^{r s}+c f_{k}^{r s}\right)} .
\end{aligned}
$$

Thus, it follows that

$$
f_{k}^{r s}=Q_{r s} \frac{e^{-\theta\left(c_{k}^{r s}+c f_{k}^{r s}\right)}}{\sum_{l \in K^{r s}} e^{-\theta\left(c_{l}^{r s}+c f_{l}^{r s}\right)}}, \quad \forall k \in K^{r s} \cup \widehat{K}^{r s}, r \in R, s \in S .
$$

Following the proof of Proposition 2, the VI formulations (22) and (23) satisfy the bimodal C-Logit SUE conditions (4)-(8).

This completes the proof.

The VI formulations (22) and (23) can be solved by selfadaptive gradient projection method [21], self-adaptive projection-auxiliary problem method [22], semismooth Newton method [23], and so on. From the proof of Proposition 2, solving the VI formulations (22) and (23) is equivalent to solving the equation system (25).

\section{Numerical Experiments}

In this section, a small-sized network is only chosen to test the bimodal C-logit SUE problem because the model has been rigorously proven in theory. The purpose of the numerical experiment is to illustrate the impact of mode split on equilibrium flow distribution under the bimodal C-logit SUE condition.

A small-sized network, as shown in Figure 2, with two OD pairs $(1,9)$ and $(3,7)$, nine nodes, and sixteen links is adopted to test the proposed model. Traffic demand for OD pairs $(1,9)$ and $(3,7)$ is 90 and 100 , respectively. The links 15 and 16 are rail links and the others are road links. The zeroflow travel cost and design capacity for each link on the network are shown in Table 2. The BPR link cost function is adopted as follows:

$$
t_{a}\left(x_{a}\right)=t_{a}^{0}\left(1+0.15\left(\frac{x_{a}}{c_{a}}\right)^{4}\right)
$$

Parameter value is assigned as $\alpha=0.2, \theta=0.5$, $\gamma=0.5, \beta=1, \quad \kappa=0.8, \quad \varepsilon_{1}=0.1, \varepsilon_{2}=0.000001, \quad \varrho=0.9$, $\sigma=500$, and $\Delta \sigma=100$.

On the general road network, Zhou et al. compared the traffic assignment results of the length-based C-logit models and the multinomial logit model on the real Winnipeg networks [14]. Their numerical results indicate that the differences between the C-logit model and the multinomial logit model are substantial and distinct, but the differences between the two logit models are relatively inconspicuous $(0.02<\mathrm{RMSE}<0.03$, for the details refer to reference [14]). In this paper, we mainly investigate the effects of the length-based C-logit SUE model on the mode split and equilibrium flow distribution and analyze the reasonable degree of the assignment results on the bimodal network since the other assignment results are discovered between the C-logit model from Zhou's numerical experiment.

In this section, a relative Evacuation Efficiency (REE, i.e., flow-capacity relationship) is adopted to capture the reasonable degree of the equilibrium flow distribution on the road network, and total travel time (TTT) is used to capture the effectiveness of the equilibrium flow distribution on the bimodal network as follows:

$$
\begin{aligned}
& \operatorname{REE}(\mathbf{x})=1-\frac{\sum_{a \in A} \max \left(0, x_{a}-c_{a}\right)}{\sum_{a \in A} x_{a}}, \\
& \operatorname{TTT}(\mathbf{x})=\sum_{a \in A \cup \widehat{A}} x_{a} t_{a}\left(x_{a}\right) .
\end{aligned}
$$

The numerical results are shown in Figure 3 by solving the bimodal C-logit SUE model.

As shown in Figure 3, on the unimode network (only the road network considered), all link flows exceed or approach to the link capacity, but only several link flows spill from the links on the bimodal network (e.g., links 3, 10, and 12). The REE is 0.8769 on the road network. The total travel time is 2657.1 on the unimode network but only 1720.6 on the bimodal one. The mode-share of OD pair $(1,9)$ and $(3,7)$ is 0.248 and 0.2841 , respectively. This means that the impact of public transport on travel demand would be underestimated severely if we only take the road network into account, which also implies that the effect of one transport mode on the other does exist and cannot be ignored in the real transportation network. 


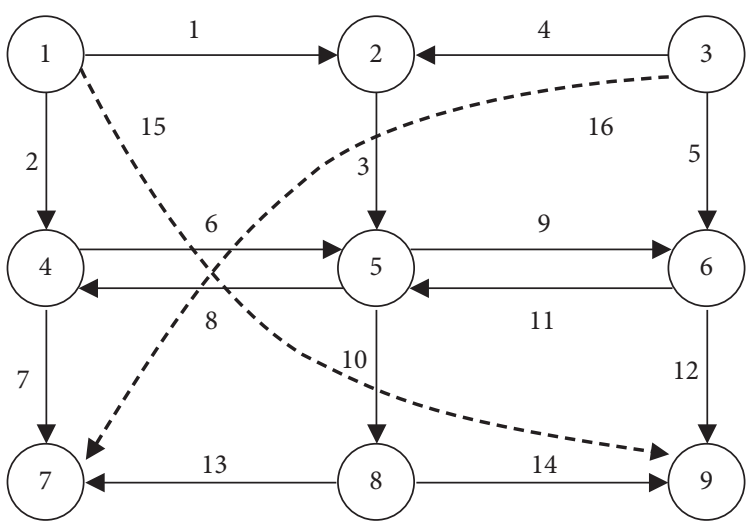

FIGURE 2: Small-sized transportation network.

TABLE 2: Link characteristics.

\begin{tabular}{|c|c|c|c|c|c|c|c|c|c|c|c|c|c|c|c|c|}
\hline Links & 1 & 2 & 3 & 4 & 5 & 6 & 7 & 8 & 9 & 10 & 11 & 12 & 13 & 14 & 15 & 16 \\
\hline$t_{a}^{0}$ & 2 & 2 & 1.5 & 2.2 & 2.2 & 1 & 2 & 1.3 & 1.5 & 1.8 & 1.5 & 2 & 2 & 2 & 12 & 12 \\
\hline$c_{a}$ & 35 & 35 & 40 & 40 & 40 & 40 & 30 & 30 & 40 & 40 & 40 & 40 & 30 & 30 & - & - \\
\hline
\end{tabular}

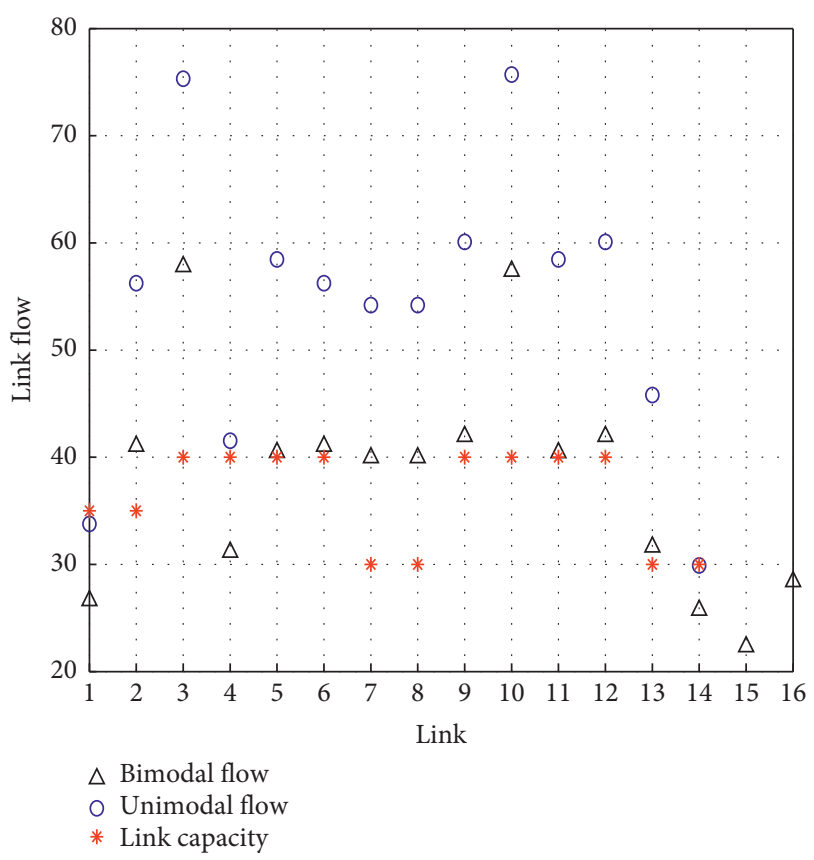

Figure 3: Equilibrium link flow distribution pattern.

\section{Conclusions and Further Studies}

This paper investigates the length-based bimodal C-logit stochastic user equilibrium problem on the bimodal transportation network with road and rail travel modes. Under the general assumptions, after redefining the link travel cost functions and employing a binary Logit model for the mode-split, the length-based model is transformed into an unconstrained nonlinear mathematical programming formulation, which is an extension of the unconstrained SUE assignment problem. The model satisfies both the
C-logit SUE and mode equilibrium conditions at its stationary point. Particularly, the stationary point is a unique link flow-based global minimum point for the strictly monotonous link cost function in the length-based bimodal C-logit SUE model. Such models can be solved easily by many existing algorithms.

In the further studies, we will investigate the second-best congestion pricing problem with the toll locations and levels on the bimodal transportation network. Furthermore, the impact of congestion pricing scheme on mode split and equilibrium flow distribution will be analyzed when the OD demand fluctuates. Traffic congestion indeed necessarily accounts for a significant share of vehicle emission and air pollution because emission may increase as vehicles spend more time in idling and undergo numerous acceleration, deceleration, and even braking [24, 25]. Congestion mitigation, environmental protection, and better travel quality provided on the road network is still an ever increasing concerned problem in the urban transportation system. Road congestion pricing has been viewed as an effective means to alleviate traffic congestion [26-29]. The fundamental principle of road pricing is to apply economic lever to travel demand management. After charging a suitable toll, traffic demand and link (path) flow can be influenced to achieve more efficient utilization of existing network.

\section{Data Availability}

The simulated data used to support the findings of this study are included within the article, and related codes are available from the corresponding author upon request.

\section{Disclosure}

Any shortcomings are our responsibility in the paper. 


\section{Conflicts of Interest}

The authors declare that they have no conflicts of interest.

\section{Acknowledgments}

This research was supported by the Natural Science Foundation of Shaanxi Province (2014JM2-1004) and Basic Foundation of Weinan Science and Technology Agency and Shaanxi Ministry of Education (2018-ZDYF-JCYJ-40 and 18JK0282).

\section{References}

[1] D.-Y. Lin, J.-H. Fang, and K.-L. Huang, "Passenger assignment and pricing strategy for a passenger railway transportation system," Transportation Letters, vol. 11, no. 6, pp. 320-331, 2019.

[2] J. G. Wardrop, "Road paper. Some theoretical aspects of road traffic research," Proceedings of the Institution of Civil Engineers, vol. 1, no. 3, pp. 325-362, 1952.

[3] C. F. Daganzo and Y. Sheffi, "On stochastic models of traffic assignment," Transportation Science, vol. 11, no. 3, pp. 253-274, 1977.

[4] C. Fisk, "Some developments in equilibrium traffic assignment," Transportation Research Part B: Methodological, vol. 14, no. 3, pp. 243-255, 1980.

[5] A. Chen, P. Kasikitwiwat, and Z. Ji, "Solving the overlapping problem in route choice with paired combinatorial logit model," Transportation Research Record: Journal of the Transportation Research Board, vol. 1857, no. 1, pp. 65-73, 2003.

[6] E. Cascetta, Transportation Systems Engineering: Theory and Methods, Kluwer Academic Publishers, Dordrecht, Netherlands, 2001.

[7] W. H. K. Lam and G. Xu, "A traffic flow simulator for network reliability assessment," Journal of Advanced Transportation, vol. 33, no. 2, pp. 159-182, 1999.

[8] Q. Meng, W. H. K. Lam, and L. Yang, "General stochastic user equilibrium traffic assignment problem with link capacity constraints," Journal of Advanced Transportation, vol. 42, no. 4, pp. 429-465, 2008.

[9] Q. Meng and Z. Liu, "Mathematical models and computational algorithms for probit-based asymmetric stochastic user equilibrium problem with elastic demand," Transportmetrica, vol. 8, no. 4, pp. 261-290, 2012.

[10] G. E. Cantarella, S. de Luca, M. Di Gangi, and R. Di Pace, "Approaches for solving the stochastic equilibrium assignment with variable demand: internal vs. external solution algorithms," Optimization Methods and Software, vol. 30, no. 2, pp. 338-364, 2015.

[11] W. Jing, I. Kim, M. Ramezani, and Z. Liu, "Stochastic traffic assignment of mixed electric vehicle and gasoline vehicle flow with path distance constraints," Transportation Research Procedia, vol. 21, pp. 65-78, 2017.

[12] S. Xu and W. Jiang, "A physarum-inspired model for the probit-based stochastic user equilibrium problem," 2017, https://arxiv.org/abs/1703.01880.

[13] E. Cascetta, F. Russo, F. A. Viola, and A. Vitetta, "A model of route perception in urban road networks," Transportation Research Part B: Methodological, vol. 36, no. 7, pp. 577-592, 2002.
[14] Z. Zhou, A. Chen, and S. Bekhor, "C-logit stochastic user equilibrium model: formulations and solution algorithm," Transportmetrica, vol. 8, no. 1, pp. 17-41, 2012.

[15] A. Chen, S. Pravinvongvuth, X. Xu, S. Ryu, and P. Chootinan, "Examining the scaling effect and overlapping problem in logit-based stochastic user equilibrium models," Transportation Research Part A: Policy and Practice, vol. 46, no. 8, pp. 1343-1358, 2012.

[16] X. Xu and A. Chen, "C-logit stochastic user equilibrium model with elastic demand," Transportation Planning and Technology, vol. 36, no. 5, pp. 463-478, 2013.

[17] Z. Zhou, A. Chen, and S. C. Wong, "Alternative formulations of a combined trip generation, trip distribution, modal split, and trip assignment model," European Journal of Operational Research, vol. 198, no. 1, pp. 129-138, 2009.

[18] C. Xie and S. Travis Waller, "Stochastic traffic assignment, Lagrangian dual, and unconstrained convex optimization," Transportation Research Part B: Methodological, vol. 46, no. 8, pp. 1023-1042, 2012.

[19] Y. Sheffi, Urban Transportation Network: Equilibrium Analysis with Mathematical Programming Methods, pp. 320-322, Prentice-Hall Englewood Cliff, Upper Saddle River, NJ, USA, 1985.

[20] M. S. Bazaraa, H. D. Sherali, and L. M. Shetty, Nonlinear Programming: Theory and Algorithms, John Wiley and Sons, New York, NY, USA, 2006.

[21] A. Chen, Z. Zhou, and X. Xu, "A self-adaptive gradient projection algorithm for the nonadditive traffic equilibrium problem," Computers \& Operations Research, vol. 39, no. 2, pp. 127-138, 2012.

[22] G. Qian, D. Xu, L. Xu, and H. Yang, "Solving nonadditive traffic assignment problems: a self-adaptive projection-auxiliary problem method for variational inequalities," Journal of Industrial \& Management Optimization, vol. 9, no. 1, pp. 255-274, 2013.

[23] M. Xu, A. Chen, Y. Qu, and Z. Gao, “A semismooth Newton method for traffic equilibrium problem with a general nonadditive route cost," Applied Mathematical Modelling, vol. 35, no. 6, pp. 3048-3062, 2011.

[24] L. Chen and H. Yang, "Managing congestion and emissions in road networks with tolls and rebates," Transportation Research Part B: Methodological, vol. 46, no. 8, pp. 933-948, 2012.

[25] O. I. Kolak, O. Feyzioglu, F. I. Birbil, N. Noyan, and S. Yalcindag, "Using emission functions in modeling environmentally sustainable traffic assignment policies," Journal of Industrial and Management Optimization, vol. 9, pp. 341363, 2013.

[26] H. Yang and H. J. Huang, Mathematica and Economic Theory of Road Pricing, Elsevier, Berlin, Germany, 2005.

[27] M. Xu, G. Wang, S. Grant-Muller, and Z. Gao, "Joint road toll pricing and capacity development in discrete transport network design problem," Transportation, vol. 44, no. 4, pp. 731-752, 2017.

[28] Y. Ye and H. Wang, "Optimal design of transportation networks with automated vehicle links and congestion pricing," Journal of Advanced Transportation, vol. 2018, pp. 1-12, 2018.

[29] G. Wang, Z. Gao, M. Xu et al., "Integrating link-based discrete credit charging scheme into discrete network design problem," European Journal of Operational Research, vol. 272, no. 1, pp. 176-187, 2019. 